\title{
Snapshots of fireballs at freeze-out from heavy-ion collisions at different energies
}

\author{
Ivan Melo*i \\ Žilinská Univerzita, Akademická 1, 01026 Žilina, Slovakia \\ E-mail: melo@fyzika.uniza.sk \\ Boris Tomášik ${ }^{1,2}$ \\ ${ }^{1}$ Univerzita Mateja Bela, Tajovského 40, 97401 Banská Bystrica, Slovakia \\ ${ }^{2}$ Czech Technical University in Prague, FNSPE, Břehová 7, 11519 Prague, Czechia \\ E-mail: boris.tomasik@umb.sk
}

European Physical Society Conference on High Energy Physics - EPS-HEP2019 -

10-17 July, 2019

Ghent, Belgium

\footnotetext{
* Speaker.

$\dagger$ We gratefully acknowledge financial support by VEGA 1/0348/18 (Slovakia), by the Czech Science Foundation via grant No. 17-04505S, and by the Ministry of Education of the Slovak Republic via project FEPO. Computing was performed in the High Performance Computing Center of the Matej Bel University in Banská Bystrica using the HPC infrastructure acquired in project ITMS 26230120002 and 26210120002 (Slovak infrastructure for high-performance computing) supported by the Research \& Development Operational Programme funded by the ERDF.
} 


\section{Introduction}

The purpose of the RHIC Beam Energy Scan (BES) programme is to study the dependences of various observables on the heavy-ion collision energy. The ultimate goal is to explore the QCD phase diagram and possibly find the critical point of the deconfinement phase transition.

The bulk evolution of the fireball is reflected in the distributions of hadrons emitted at the point of their kinetic freeze-out. By combining information from single-particle spectra of different sorts of hadrons it is then possible to reconstruct this freeze-out state, in particular its temperature and the transverse expansion velocity. Since the knowledge of the final state allows us (in principle) to deduce the previous evolution and the properties of the matter, it is interesting to perform these studies at different collision energies.

In this contribution we fit the measured single-particle spectra of pions, kaons, and (anti)protons from central $\mathrm{Au}+\mathrm{Au}$ or $\mathrm{Pb}+\mathrm{Pb}$ collisions in the energy range $\sqrt{s_{N N}}=7.7$ to $2760 \mathrm{GeV}$ with the predictions of the blast wave model with an included production of resonances. We assume the partial chemical equilibrium which develops if the chemical composition freezes out at the hadronisation but the fireball stays together until the kinetic freeze-out. The consequence is that although the effective numbers of the final state hadrons are constant, their production mode moves away from the resonance production to the direct production with the decreasing temperature. The predicted spectra were generated with DRAGON—a Monte Carlo generator of the final state hadrons [1, 2].

There are other similar analyses published, some of them without the resonance contribution $[3,4,5,6]$, others in the context of the Cracow single freeze-out model $[7,8,9,10]$. We have previously fitted the spectra from ALICE in [11] with the model used here, but without the assumption of the partial chemical equilibrium. Recently a similar paper has appeared, in which the spectra from ALICE are fitted with the blast-wave model [12]. Resonance decays are included there with the help of a new treatment introduced in [13].

Our results show that the fireball freezes out at a lower temperature and a stronger transverse expansion if the collision energy is increased. The spectra with the full resonance production are very similar to those calculated for only directly produced particles at higher energies.

We start with a brief explanation of the model and the experimental data. In Section 4 we present our results and in Section 5 we discuss individual contributions to the spectra from the decays of resonances.

\section{The model}

Our theoretical treatment of the single particle spectra is based on the well-known blast wave model $[14,15]$, embedded in the Monte Carlo generator DRAGON. The model is given by its emission function for hadrons of the type $i$ :

$$
\begin{gathered}
S(x, p) d^{4} x=g_{i} \frac{m_{t} \cosh (\eta-y)}{(2 \pi)^{3}}\left(\exp \left(\frac{p_{\mu} u^{\mu}-\mu_{i}}{T}\right)+s_{i}\right)^{-1} \theta\left(1-\frac{r}{R}\right) \\
\times r d r d \varphi \delta\left(\tau-\tau_{0}\right) \tau d \tau d \eta .
\end{gathered}
$$

here $\tau=\sqrt{t^{2}-z^{2}}$ is the longitudinal proper time and $\eta=\frac{1}{2} \ln ((t+z) /(t-z))$ the space-time rapidity. In the transverse plane, polar coordinates $r, \varphi$ are used. Furthemore, $T$ is temperature, $m_{t}$ 
transverse mass and $\mu_{i}$ chemical potential. The quantum statistical distributions are given by $s_{i}=1$ $(-1)$ for fermions (bosons) and $g_{i}$ is the spin degeneracy. The freeze-out time does not depend on radial coordinate, but it does depend on the longitudinal coordinate implicitly via $\tau=\tau_{0}$, i.e. $t=\sqrt{\tau_{0}^{2}+z^{2}}$. Density is distributed uniformly within the radius $R$. The transverse expansion velocity is given by

$$
v_{t}=\tanh \eta_{t}=\eta_{f}\left(\frac{r}{R}\right)^{n}
$$

The parameter $\eta_{f}$ parametrises the transverse flow gradient and $n$ the profile of the transverse velocity. The mean transverse velocity is then

$$
\left\langle v_{t}\right\rangle=\frac{2}{n+2} \eta_{f} .
$$

The transverse size $R$ and the freeze-out proper time $\tau_{0}$ influence total normalisations of transverse momentum spectra and also influence the sizes of the HBT radii. A fit which obtains $R$ and $\tau_{0}$ should thus also take into account the data on HBT radii. Due to complexity of such a problem we chose not to embark on it now and remain without the sensitivity to these geometrical parameters.

Resonances are emitted as described by the emission function in eq. (2.1) and then decay exponentially in time with the mean lifetime given by the inverse of the resonance width. The decay is determined by the phase-space only. We include baryon resonances up to the mass of $2 \mathrm{GeV}$ and mesonic resonances up to $1.5 \mathrm{GeV}$.

We assume two distinct freeze-outs: the chemical one with the chemical freeze-out temperature $T_{c h}$ and the kinetic freeze-out at $T=T_{k i n}$ much lower than $T_{c h}$. At the chemical freeze-out, inelastic collisions become rare and the system gets out of the full chemical equilibrium. Nevertheless, since the abundances of the final state stable species are fixed, each species develops a nonzero temperature-dependent chemical potential $\mu_{i}$. At this phase interactions still maintain the partial chemical equilibrium between lowest state stable hadrons and resonances through which they interact [16]. Also, elastic collisions keep the local thermal equilibrium until the fireball freezes out completely. Resonances are then let to decay so that in the end we look only at stable hadrons. The calculated chemical potentials as functions of temperature were tabulated [17] and read into DRAGON as input in the simulations.

\section{The fitting procedure}

The transverse momentum spectra of (anti)protons, pions and kaons from the most central heavy ion collisions $\left(0-6 \%\right.$ for $\sqrt{s_{N N}}=130 \mathrm{GeV}, 0-5 \%$ for all other energies) were measured by the STAR and ALICE collaborations: $\mathrm{Au}+\mathrm{Au}$ at $\sqrt{s_{N N}}=7.7,11.5,19.6,27$, and $39 \mathrm{GeV}$ [4], $\mathrm{Au}+\mathrm{Au}$ at $\sqrt{s_{N N}}=62.4,130,200 \mathrm{GeV}$ [5], and $\mathrm{Pb}+\mathrm{Pb}$ at $\sqrt{s_{N N}}=2760 \mathrm{GeV}$ [3].

The theoretical spectra were generated by DRAGON in 400 training points in the threeparameter space (freeze-out temperature $T$, transverse flow gradient $\eta_{f}$, profile of the transverse velocity $n$ ). The typical run-time for one training point is $30 \mathrm{~min}$ to 5 hours in order to generate enough events to suppress the MC statistical errors well below the combined experimental uncertainties. Then the spectra were normalised to match the normalisation of the measured spectra, species by species, i.e. six independent normalisations. 
We set the upper value of the fitted $p_{t}$ interval to $2 \mathrm{GeV}$ for $\sqrt{s_{N N}}=7.7-39 \mathrm{GeV}$ (the upper end of the measured $p_{t}$ range) and also for the ALICE spectra (here the motivation is to remain in the region sensitive to collective effects). For $\sqrt{s_{N N}}=62.4-200 \mathrm{GeV}$, the measured/published $p_{t}$ spectra are shorter, reaching to $0.7-1.1 \mathrm{GeV}$, according to the species. The lower end of the fitted intervals coincides with the lower end of the measured $p_{t}$ range. The only exception (to be discussed later) are the ALICE pion $p_{t}$ spectra - the data start at $p_{t}=100 \mathrm{MeV}$ while the fit starts at $p_{t}=250 \mathrm{MeV}$.

For the data fitting we have used the MADAI statistical analysis package [18]. MADAI performs the Markov Chain Monte Carlo exploration of the parameter space using the GaussianProcess based emulator trained on the training points. The output of MADAI is the best fit point in our three-parameter space. As a final step (and a cross-check of MADAI) we calculated the $\chi^{2}$ value at the best fit point.

\section{Results}

The main results of this contribution are the energy dependences of the kinetic freeze-out temperature $T$, the transverse flow gradient $\eta_{f}$, the profile of the transverse velocity $n$, and the mean transverse velocity $v_{t}=2 \eta_{f} /(n+2)$, shown in Fig. 1 . Three sets of points are plotted: the full results obtained from the fits to spectra up to $2 \mathrm{GeV}$ with the contribution from the resonance decays (solid red circles), results from the fits to spectra up to $2 \mathrm{GeV}$ without the resonance contribution (blue squares) and results from the fits to short (cut down) $p_{t}$ spectra up to $\sim 1 \mathrm{GeV}$ which match the shorter $p_{t}$ intervals for $\sqrt{s_{N N}}=62,130,200 \mathrm{GeV}$ with the resonance contribution included (empty black circles). In Table 1 we summarize the numerical values of the best fit parameters for the full results together with the $\chi^{2} / n_{d o f}$ value.

Table 1: Parameters of the best fits for different energies. These parameters were used also for the calculation of the resonance composition of the transverse momentum spectra.

\begin{tabular}{|c|cccc|}
\hline$\sqrt{s_{N N}}[\mathrm{GeV}]$ & $T[\mathrm{MeV}]$ & $\eta_{f}$ & $n$ & $\chi^{2} / n_{\text {dof }}$ \\
\hline 7.7 & $102.0 \pm 2.0$ & $0.620 \pm 0.016$ & $0.726 \pm 0.073$ & 0.83 \\
11.6 & $103.6 \pm 1.5$ & $0.632 \pm 0.012$ & $0.792 \pm 0.069$ & 0.66 \\
19 & $98.1 \pm 1.6$ & $0.711 \pm 0.009$ & $1.122 \pm 0.064$ & 0.38 \\
27 & $97.4 \pm 1.4$ & $0.715 \pm 0.007$ & $1.022 \pm 0.048$ & 0.68 \\
39 & $98.5 \pm 1.4$ & $0.729 \pm 0.007$ & $1.006 \pm 0.045$ & 0.47 \\
62 & $80.2 \pm 0.8$ & $0.756 \pm 0.007$ & $0.689 \pm 0.020$ & 0.93 \\
130 & $75.0 \pm 0.8$ & $0.797 \pm 0.006$ & $0.760 \pm 0.015$ & 1.07 \\
200 & $75.4 \pm 1.8$ & $0.841 \pm 0.012$ & $0.810 \pm 0.020$ & 0.25 \\
2760 & $78.3 \pm 1.6$ & $0.903 \pm 0.005$ & $0.766 \pm 0.018$ & 0.32 \\
\hline
\end{tabular}

The kinetic freeze-out temperature starts at $T \sim 100-104 \mathrm{MeV}$ at the lowest energies and decreases to $T \sim 75-80 \mathrm{MeV}$ at the highest energies. There is a small discontinuity between $\sqrt{s_{N N}}=39$ and $62.4 \mathrm{GeV}$ (seen in full results, solid red circles). This is likely due to the difference in the fitted $p_{t}$ range between the two energies. Indeed, once the $p_{t}$ intervals are matched (empty 

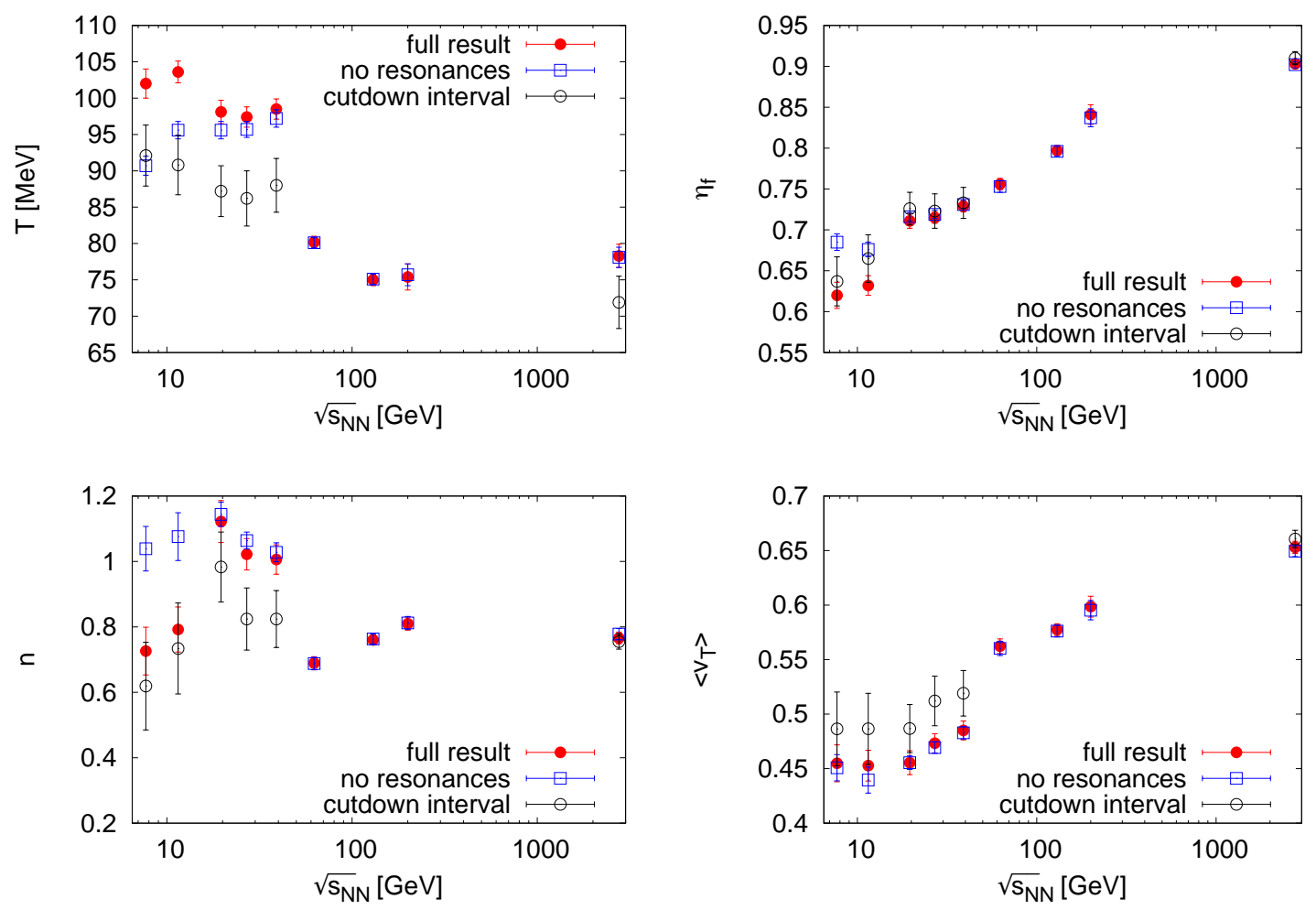

Figure 1: Energy dependence of the freeze-out parameters: temperature (upper left), transverse flow gradient (upper right), exponent of the transverse velocity profile (lower left), mean transverse velocity (lower right).

cles), the discontinuity is suppressed. The impact of resonances is very small at high energies while at the lowest energies they lead to an upward shift in the temperature of about $10 \mathrm{MeV}$. The reason is the lower population of particles from resonance decays and their uniform $p_{t}$ distribution at high energies. The transverse flow gradient increases from $\eta_{f}=0.62$ at $\sqrt{s_{N N}}=7.7 \mathrm{GeV}$ to $\eta_{f}=0.90$ at $\sqrt{s_{N N}}=2760 \mathrm{GeV}$. The effect of resonances is again negligible except for the two lowest energies. The mean transverse velocity increases with energy from $v_{t}=0.45$ to $v_{t}=0.65$ while the profile of the transverse velocity $n$ fluctuates as the least sensitive parameter.

The simulated transverse momentum spectra of $\pi^{+}$corresponding to the best fit parameters (full results in Table 1) are shown in Fig. 2 for different energies as solid lines along with the measured data points (statistical and systematic uncertainties added in quadrature) from STAR and ALICE. The spectra of protons and kaons were published in our paper [17].

The quality of the fits, excellent for all energies, is violated by the low $p_{t}$ pions at $\sqrt{s_{N N}}=$ $2760 \mathrm{GeV}$ where Monte Carlo underestimates the data and the first six $p_{t}$ bins were excluded from the fit. This disagreement with the data was shown to disappear within the context of a chemical non-equilibrium version of the statistical hadronization model [7]. Note, however, that the enhancement of the pion spectrum at low $p_{t}$ may well be caused by a specific shape of the freeze-out hypersurface in this model in which the matter at larger distance from the longitudinal axis of the fireball freezes-out later than the matter in the middle. 


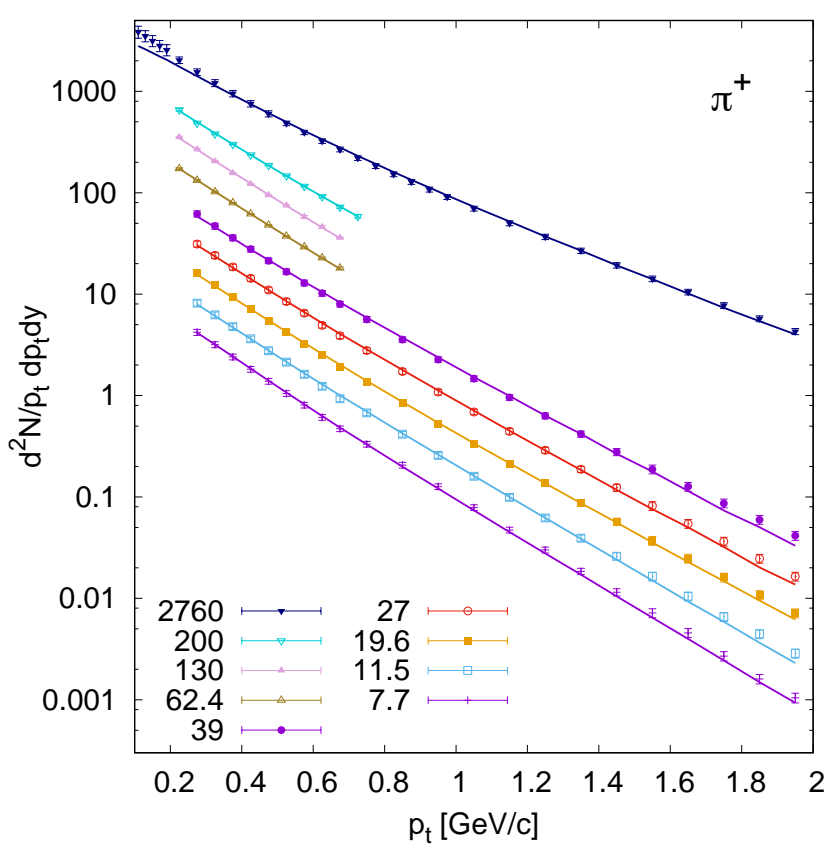

Figure 2: Transverse momentum spectra of $\pi^{+}$for different energies. To display all spectra in one figure we divide data for $\sqrt{s_{N N}}=7.7,11.5,19.6,27,39,62.4,130,200,2760 \mathrm{GeV}$ by factors $256,128,64,32,16,8,4,2$ and 0.5 , respectively.

\section{Composition of the spectra according to the origin of the pions}

An important part of the final state pions (results for protons and kaons were published in [17]) comes from the decays of resonances. In Dragon we can track the origin of pions and thus decipher the anatomy of the spectra. The results, calculated with the parameters from Table 1 for four chosen energies, $\sqrt{s_{N N}}=7.7,11.5,27,200 \mathrm{GeV}$, are plotted in Figure 3 .

We observe that the fraction of resonance-produced pions decreases as the collision energy goes up. This behaviour is connected with the partial chemical equilibrium. The relative resonance production is high at the chemical freeze-out. Afterwards, however, the temperature decreases and the weight of the production moves from the resonances towards the directly produced pions.

Further, at the lower energies the resonances tend to populate the low $p_{t}$ area while at the $200 \mathrm{GeV}$ the share of the resonance production is rather flat as a function of $p_{t}$. A closer inspection reveals that the feature is mainly brought in by the $\Delta$ resonance. Its decay is close above the threshold, so that daughter particles do not acquire a high momentum. In combination with the small transverse expansion velocity this causes that the pions from such decays stay at low $p_{t}$.

Let us also stress that at the lower energies the contribution from the baryon resonances (most importantly $\Delta$ ) to the pion production is more important than at the higher energies.

\section{Conclusions}

Our results show that the fireball develops stronger transverse expansion and cools down fur- 

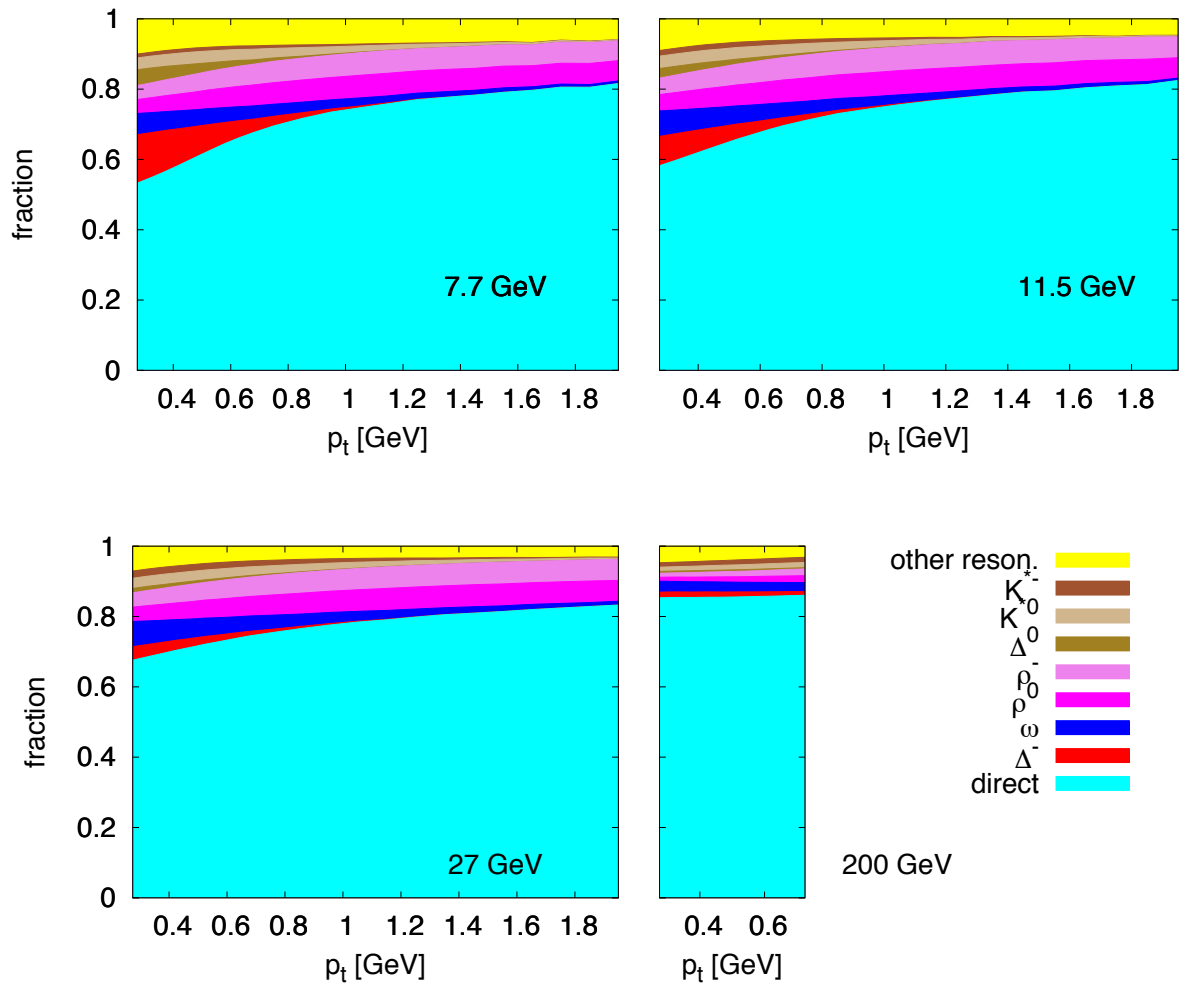

Figure 3: The composition of the $p_{t}$ spectra of $\pi^{-}$at $\sqrt{s_{N N}}=7.7,11.5,27$ and $200 \mathrm{GeV}$. Plotted are the ratios of $\pi^{-}$of a given origin (direct or from a particular resonance) to the total number of $\pi^{-}$as functions of $p_{t}$. The bands from bottom show the relative contributions from direct pions and pions from $\Delta^{-}, \omega, \rho^{0}$, $\rho^{-}$and other resonances.

ther with the increase of the collision energy. The latter decrease of the kinetic freeze-out temperature by more than $20 \mathrm{MeV}$ can be understood: more energy and entropy is deposited which expands to a larger volume. At the larger volume the local longitudinal flow gradients are smaller and so is the expansion rate. Thus, the interaction rate at the freeze-out drops lower, and so does the temperature.

Although the kinetic freeze-out temperature seems to show a sharp discontinuity between 39 and $62.4 \mathrm{GeV}$, the feature may be connected with the different coverage of the $p_{t}$ intervals in the different data sets.

It is interesting to observe that the full results obtained with the inclusion of the resonances coincide with those obtained with only directly produced hadrons. This is seen for all but the two lowest collision energies. The cooling between the chemical and the kinetic freeze-out temperatures is most pronounced at the high collision energies where the temperature drops from about $160 \mathrm{MeV}$ to some $80 \mathrm{MeV}$, i.e. by $80 \mathrm{MeV}$. In contrast to that, at $\sqrt{s_{N N}}=7.7 \mathrm{GeV}$ it went down only by roughly $40 \mathrm{MeV}$ between 144 and $102 \mathrm{MeV}$. In view of these numbers it is clearly understood that the influence of the resonances becomes less important at the high energies.

In this paper we presented the results of a pilot study where only data from central collisions have been fitted. A more comprehensive study which will also include the centrality dependence is being elaborated and will be published later. 


\section{References}

[1] B. Tomášik, Comput. Phys. Commun. 180, 1642 (2009) [arXiv:0806.4770 [nucl-th]].

[2] B. Tomášik, Comput. Phys. Commun. 207 (2016) 545. doi:10.1016/j.cpc.2016.06.011

[3] B. Abelev et al. [ALICE collaboration], Phys. Rev. C 88, 044910 (2013).

[4] L. Adamczyk et al. [STAR collaboration], Phys. Rev. C 96, 044904 (2017).

[5] B.I. Abelev et al. [STAR collaboration], Phys. Rev. C 79, 034909 (2009).

[6] L. L. Li and F. H. Liu, Eur. Phys. J. A 54 (2018) no.10, 169 doi:10.3847/1538-4365/aada4a, 10.1140/epja/i2018-12606-3 [arXiv:1809.03881 [hep-ph]].

[7] V. Begun, W. Florkowski and M. Rybczyński, Phys. Rev. C 90, no. 1, 014906 (2014) [arXiv:1312.1487 [nucl-th]].

[8] V. Begun, W. Florkowski and M. Rybczyński, Phys. Rev. C 90, no. 5, 054912 (2014) [arXiv:1405.7252 [hep-ph]].

[9] D. Prorok, J. Phys. G 43 (2016) no.5, 055101 doi:10.1088/0954-3899/43/5/055101 [arXiv:1508.07922 [nucl-th]].

[10] D. Prorok, Eur. Phys. J. A 55 (2019) 37 doi:10.1140/epja/i2019-12709-3 [arXiv:1804.05691 [hep-ph]].

[11] I. Melo and B. Tomasik, J. Phys. G 43 (2016) no.1, 015102 doi:10.1088/0954-3899/43/1/015102 [arXiv:1502.01247 [nucl-th]].

[12] A. Mazeliauskas and V. Vislavicius, [arXiv:1907.11059 [hep-ph]].

[13] A. Mazeliauskas, S. Floerchinger, E. Grossi and D. Teaney, Eur. Phys. J. C 79 (2019) no.3, 284 doi:10.1140/epjc/s10052-019-6791-7 [arXiv:1809.11049 [nucl-th]].

[14] P. J. Siemens and J. O. Rasmussen, Phys. Rev. Lett. 42, 880 (1979).

[15] E. Schnedermann, J. Sollfrank and U. Heinz, Phys. Rev. C 48, 2462 (1993) [nucl-th/9307020].

[16] H. Bebie, P. Gerber, J. L. Goity and H. Leutwyler, Nucl. Phys. B378 (1992) 95. doi:10.1016/0550-3213(92)90005-V

[17] I. Melo and B. Tomasik, Kinetic freeze-out in central heavy-ion collisions between 7.7 and $2760 \mathrm{GeV}$ per nucleon pair, e-Print: arXiv:1908.03023.

[18] J. Novak, K. Novak, S. Pratt, C. E. Coleman-Smith and R. L. Wolpert, arXiv:1303.5769 [nucl-th] (2013). http://arxiv.org/abs/1303.5769 